\title{
Tangible Bits and Malleable Atoms in the Design of a Computer Music Instrument
}

\author{
Roel Vertegaal \\ Queen's University \\ Canada \\ roel@acm.org
}

\begin{abstract}
We present SensOrg, a computer music instrument designed as a modular assembly of input/output devices and musical software, mapped and arranged according to functional characteristics of the musician-instrument system. Using tangible bits and malleable atoms, we externally represented the musical software functionality in a physical interface which is freezable yet totally flexible.
\end{abstract}

KEYWORDS Tangible Bits, Malleable Atoms, Affective Computing, Musical Instrument Design, Computer Music.

\section{INTRODUCTION}

Computer music instruments are musical instruments in which a computer is used to generate musical audio, on the basis of electronic input signals provided by a human performer. Unlike traditional musical instruments, computer music instruments are not governed by the real-world physics of the sound generation process. Designers of computer music instruments therefore enjoy the freedom to uncouple the physical input from the physical output. Such malleability of input devices and sound production processes has resulted in the use of interesting new control structures, and allowed a creative randomness in the control of musical sounds. However, we believe the freedom of information structure associated with computer music instrument design has problems of its own. Most importantly, this freedom may result in a mismatch of human and computer inputoutput modalities [3]. Musicians need to achieve an extraordinarily sophisticated level of nonverbal, emotional, expression. Their skills involve heavy sensory-motor processing, a requirement that has influenced the design and utilization of traditional musical instruments for centuries. We believe traditional instruments are more usable than computer music instruments, for three reasons:

(1) They provide tangible feedback of the state of the musical activity.

(2) They provide tangible input of the muscle tension patterns that represent human emotion [1].

(3) They tend to not change, supporting training and predictable performance.

With his Hyperinstruments, Machover [2] tried to address the above problem by augmenting traditional instruments with computer input devices, thus combining the tangibility of the former with the malleability of the latter. However, we feel this was a circumvention rather than a

\author{
Tamas Ungvary \\ Royal Institute of Technology \\ Stockholm, Sweden \\ ungvary@kth.se
}

solution of the problem. In [3], we proposed the new paradigm of Cyberinstruments, which essentially consist of an array of tangible computer input devices that can be flexibly arranged so as to represent the hidden musical software functionality. Although SensOrg, our first Cyberinstrument, looks like an organ or percussion kit, it is more like a musician's cockpit (see Figure 1). In its design, we approached the musician-instrument system as a whole, rather than as a set of simple input-output relationships. Elements include: symbolic and nonverbal intent of the musician or composer, human actuator channels, I/O devices, software functionality and human perceptual channels, with information flowing between elements from human to instrument and back. We first will discuss how musical timing constraints influenced the tangibility and malleability of our design, after which we present our design rationale around a discussion of the interaction devices.

TANGIBILITY, MALLEABILITY AND TIME

Making music is a real-time business. During performance or improvisation, musicians have little time for higher-order cognitive processing. Performance interfaces should therefore allow the development of motor skills, as well as address human information channels that convey the musical intent as directly as possible. The advantages of interfacing with the human motor system through tactilekinesthetic channels are clear: (a) the muscle tension they convey forms a direct representation of the nonverbal expressions to be communicated through the instrument [1], and (b) they are fast and require little semantic processing. However, instruments need also be configured for use. This is particularly true for computer music instruments, in which the arrangement of sounds and I/O mappings form an important part of the compositional process. Here, time constraints are much more relaxed, allowing the use of more symbolic interfaces (e.g., musical scores). Throughout the design, we associated tangibility with the more real-time nonverbal performance functionality and malleability with the more relaxed symbolic compositional functionality.

\section{DESIGN RATIONALE}

\section{Tangible Input for Efficient Performance}

First, we organized the input devices in SensOrg according to the feedback characteristics associated with their use [3]. We used visual feedback for the more relaxed symbolic functions, and auditory, tactile-kinesthetic feedback for the more real-time nonverbal functions. We applied buttons, faders, touch screen and mouse for the more relaxed symbolic functions, and buttons, faders, trackballs and isometric joysticks for the more real-time nonverbal functions [3]. For the performance modalities of the 


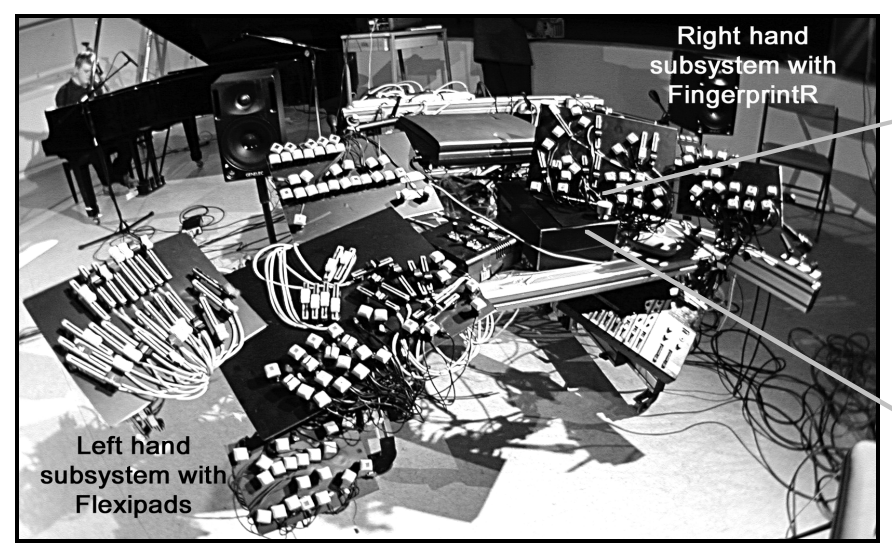

Figure 1. SensOrg with non-dominant part left and dominant instrument, we tried to introduce as much explicit knowledge into the real world as possible, reducing requirements for knowledge in the head of the musician. Essentially, we tried to externally represent the state of internal software functionality as tangible bits. All input devices can be frozen into a unique spatial arrangement, according to their musical functionality. Each device is coded by color, shape, orientation within groupings and textual information. SensOrg is divided into two parts: one for the dominant hand, and one for the non-dominant hand. The dominant hand exercises the more real-time nonverbal functions, while the non-dominant hand exercises the more relaxed symbolic functions. In the center of the dominant hand is the Fingerprint $R$, a 3D isometric joystick that conveys states of tension as exerted by subtle changes in force (Figure 2) [1]. This is the most important device for the real-time nonverbal modality. To provide optimal haptic feedback, its knob follows the form of the finger with which it is played. Around the dominant hand, pads with buttons are arranged such that they can be pressed while playing the FingerprintR (Figure 2).

\section{Malleable Atoms for Flexible Configuration}

We will now discuss how we made the system flexible, so that it could be adapted to different individuals and compositional uses. We could only choose to reflect the state of internal software functionality in the external devices if we also reflected the malleability of software functionality in the external devices. If the software functionality changes, the external devices should change and vice versa. We did this by taking a modular approach to both software functionality and hardware devices. Each hardware module is connected to software functions running in Max on a PowerMac [3]. The software modules can be configured in a relaxed, symbolic fashion by means of a graphical user interface. They can be driven in a real-time nonverbal fashion by playing the corresponding hardware modules. Similarly, hardware modules can be configured in a more relaxed symbolic fashion by mapping them onto a software module, labeling them with a concept describing functionality (with the device type being a label by itself), coloring them, positioning them freely within groups, and orienting groups freely within the instrument. They can be configured in a more real-time, nonverbal fashion by selecting predefined configurations of software mappings using predefined buttons. As such, SensOrg hardware is

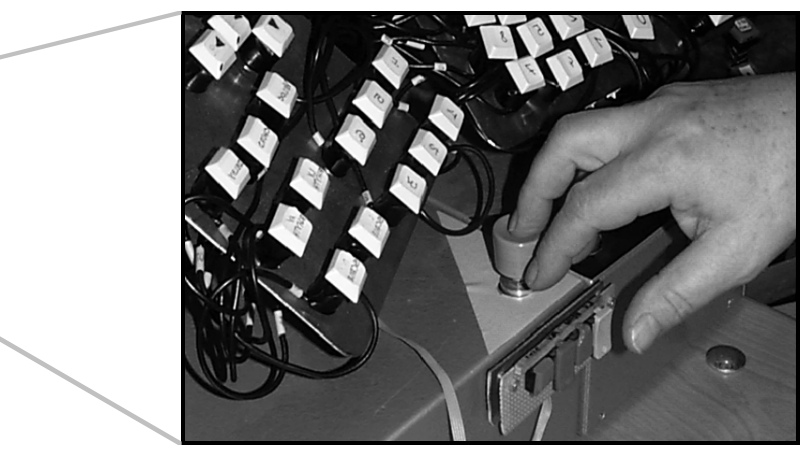

Figure 2. FingerprintR.

almost totally adaptable to circumstances. The area covered by the non-dominant hand is much larger than that of the dominant hand. In the center of this area are groupings of faders and buttons. These are the most important devices for the more relaxed symbolic functions of the performance instrument. Button and fader modules stick to a position on a metal pad by means of small magnets. These pads (called Flexipads) can be positioned and oriented freely in space, and button and fader modules can be freely positioned on the pad (Figure 3). Fader modules can be grouped so that they can be operated simultaneously with one hand gesture. Fader modules and button arrangements can be fitted to the hand by putting the hand onto a selection of devices, and then molding the devices around the contour of the hand. Malleability is also demonstrated by Image-in-Kit, an active desk used to display graphic compositions. The position of virtual buttons on its touch-sensitive surface can be associated with graphic elements of the artwork. Thus, recall of the buttons' functionality can be improved.

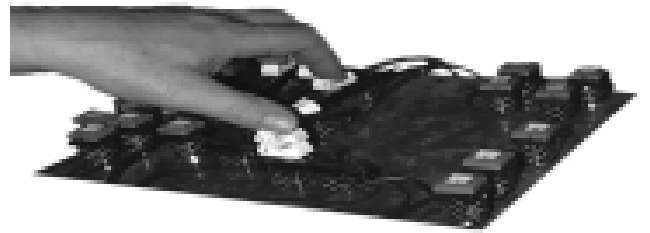

Figure 3. Flexipad with magnetic buttons and faders. CONCLUDING REMARKS

Figure 1 shows how the discussed hardware modules fit together in SensOrg. Devices are mounted on gimbals attached to a rack with adjustable arms, allowing them to be placed at any position or orientation. On the left, we see the non-dominant hand sub-system, with several Flexipads. On the Flexipads, modular structures of faders and buttons are shown. On the right of the figure, we see the dominant hand sub-system with the FingerprintR in the middle, and two small Flexipads with real-time functionality around it.

\section{REFERENCES}

1. Clynes, M. Toward a View of Man. In Biomedical Engineering Systems. NY: McGraw-Hill, 1970.

2. Machover, T. Classic Hyperinstruments. MIT Media Lab Research Report, 1992.

3. Ungvary, T. and Vertegaal, R. SensOrg: Time-complexity and the Design of a Musical Cyberinstrument. In Proceedings of ICMC'99. Beijing, China: ICMA, 1999. 